\title{
Self-perceived long-term transfer of learning after postpartum hemorrhage simulation training
}

Citation for published version (APA):

Pinto de Melo, B. C., Falbo, A. R., Sorensen, J. L., van Merrienboer, J. J. G., \& van der Vleuten, C. (2018). Self-perceived long-term transfer of learning after postpartum hemorrhage simulation training. International Journal of Gynecology \& Obstetrics, 141(2), 261-267. https://doi.org/10.1002/ijgo.12442

Document status and date:

Published: 01/05/2018

DOI:

10.1002/ijgo.12442

Document Version:

Publisher's PDF, also known as Version of record

\section{Document license:}

Taverne

\section{Please check the document version of this publication:}

- A submitted manuscript is the version of the article upon submission and before peer-review. There can be important differences between the submitted version and the official published version of record.

People interested in the research are advised to contact the author for the final version of the publication, or visit the DOI to the publisher's website.

- The final author version and the galley proof are versions of the publication after peer review.

- The final published version features the final layout of the paper including the volume, issue and page numbers.

Link to publication

\footnotetext{
General rights Owners
rights.

- You may freely distribute the URL identifying the publication in the public portal. please follow below link for the End User Agreement:

www.umlib.nl/taverne-license

Take down policy

If you believe that this document breaches copyright please contact us at:

repository@maastrichtuniversity.nl

providing details and we will investigate your claim.
}

Copyright and moral rights for the publications made accessible in the public portal are retained by the authors and/or other copyright owners and it is a condition of accessing publications that users recognise and abide by the legal requirements associated with these

- Users may download and print one copy of any publication from the public portal for the purpose of private study or research.

- You may not further distribute the material or use it for any profit-making activity or commercial gain

If the publication is distributed under the terms of Article $25 \mathrm{fa}$ of the Dutch Copyright Act, indicated by the "Taverne" license above, 


\title{
Self-perceived long-term transfer of learning after postpartum hemorrhage simulation training
}

\author{
Brena Carvalho Pinto de Melo ${ }^{1,2,3, *} \mid$ Ana Rodrigues Falbo ${ }^{4,5}$ | Jette Led Sorensen ${ }^{6}$ | \\ Jeroen J.G. van Merriënboer ${ }^{3}$ | Cees van der Vleuten ${ }^{3}$
}

${ }^{1}$ Curso de Medicina, Faculdade Pernambucana de Saúde, Recife, Brazil

${ }^{2}$ Centro de Atenção à Mulher, Instituto de Medicina Integral Professor Fernando Figueira, Recife, Brazil

${ }^{3}$ School of Health Professions

Education, Faculty of Health, Medicine and Life Sciences, Maastricht University,

Maastricht, Netherlands

${ }^{4}$ Faculty Development

Coordination, Faculdade Pernambucana

de Saúde, Recife, Brazil

${ }^{5}$ Diretoria de Pesquisa, Instituto de Medicina Integral Professor Fernando Figueira,

Recife, Brazil

${ }^{6}$ Juliane Marie Centre for Children, Women and Reproduction, Rigshospitalet, University of Copenhagen, Copenhagen, Denmark

\section{*Correspondence}

Brena C.P. Melo, Faculdade Pernambucana de Saúde \& Instituto de Medicina Integral Professor Fernando Figueira, Recife, Brazil.

Email: bcpmelo@gmail.com

๑ This article includes a Video abstract, available at https://youtu.be/IndvRBL89bo.

(1) This article includes a Portuguese translation of the Abstract, available in the Supporting Information section.

Funding Information

Faculdade Pernambucana de Saúde; Instituto de Medicina Integral Professor Fernando Figueira

\begin{abstract}
Objective: To explore long-term transfer (application of acquired knowledge and skills on the job) after postpartum hemorrhage simulation training based on either instructional design (ID) principles or conventional best practice.

Methods: In this qualitative study, semi-structured interviews with obstetrics and gynecology healthcare practitioners were conducted between August 7 and September 26, 2015, in Recife, Brazil. The participants were randomly selected from each of two postpartum hemorrhage simulations attended 2 years earlier (one ID and one conventional best practice). Thematic analysis was used to explore (1) residents' perceptions of long-term transfer of learning, (2) ID elements influencing the perceived long-term transfer, and (3) differences in the participants' perceptions according to the type of simulation attended.

Results: There were 12 interview participants. After either simulation format, residents perceived long-term transfer effects. Training design factors influencing transfer were, in their opinion, related to trainees' characteristics, simulation design, and workplace environment. Trainees who participated in the ID-based simulation perceived better communication skills and better overall situational awareness: "I didn't do that before."

Conclusion: All residents perceived long-term transfer after simulation training for postpartum hemorrhage. Those who attended the ID format additionally perceived improvements in communication skills and situational awareness, which are fundamental factors in the management of postpartum hemorrhage.
\end{abstract}

\section{KEYWORDS}

Communication; Instruction; Postpartum hemorrhage; Simulation training; Situational awareness; Training transfer

\section{1 | INTRODUCTION}

Postpartum hemorrhage (PPH) is the leading cause of maternal mortality worldwide, with most deaths considered preventable and attributable to human factors such as poor communication and lack of situational awareness. To improve the management of PPH, simulation training is strongly recommended. ${ }^{1-3}$ Effective training, however, relies on workplace application of the knowledge and skills acquired during training-in other words, on transfer of learning. ${ }^{4}$

Systematic reviews on the effectiveness of healthcare simulation training have recommend the implementation of instructional design (ID) principles, particularly when aiming to achieve transfer ${ }^{5,6}$; yet, these principles are sparsely adopted. Data on transfer from healthcare simulation training are scarce because of the complexity of 
measuring on-the-job performance, and studies have mainly explored the perceptions of trainees on short-term transfer (hours or weeks after training). ${ }^{7}$ Effective training, however, should also promote longterm transfer. ${ }^{8}$

The present study was conducted to explore self-perceived longterm transfer 2 years after having attended PPH simulation training based on either ID principles or current best practice (BP). The ID format applied principles derived from cognitive psychology that are explicitly aimed at optimizing long-term transfer ${ }^{9,10}$; the ID format used in the present study included multiple elements (Table 1).

The current study was designed to address the following research questions. (1) Do the residents perceive transfer 2 years after having attended PPH simulation training (ID or BP format)? (2) Which factors of the simulation training do they perceive as having positively affected long-term transfer? (3) Does the perceived long-term transfer differ according to the format (BP or ID) of the simulation training?

\section{2 | MATERIALS AND METHODS}

In the present qualitative study, semistructured interviews were conducted between August 7 and September 26, 2015, with healthcare practitioners who had attended one of two different PPH simulations (based on ID or BP) 2 years previously. ${ }^{15}$ At the time of the simulations, all attendees were residents at one of the five teaching hospitals in Recife, Brazil, in one of the 3 years of the obstetrics and gynecology program. Using a simple randomization method (masking of names), six attendees from each simulation format were selected for invitation to interview from a total of 54 attendees. The study was approved by the ethics committee of Instituto de Medicina Integral Professor Fernando Figueira, Recife, Brazil. All participants gave written informed consent.

The two PPH simulations differed with regard to the ID elements (Table 1) and the number of steps. The ID-based simulation contained eight steps: (1) prior knowledge activation, (2) video demonstration,
(3) dual-coding PPH protocol discussion, (4) training scenario \#1, (5) debriefing, (6) training scenario \#2 with immediate feedback, (7) training scenario \#3, and (8) debriefing with self-assessment. The training scenarios had an increasing level of complexity. The BP simulation contained three steps: (1) prior knowledge activation, (2) training scenario (identical to ID simulation training scenario \#3), and (3) debriefing. In both scenarios, the debriefings included participants watching video of themselves from the simulations. This format replicated the "best PPH simulation" previously ${ }^{16}$ selected by seven training experts.

The PPH management protocol was developed by a focus group of obstetricians and anesthesiologists based on best available evidence. ${ }^{1,17}$ The protocol was presented as shown in Figure 1, in agreement with Paivio's dual-coding theory, ${ }^{12}$ which states that content presented via a combination of text and pictures is easier to remember than content presented with either method alone.

In both simulation formats, the scenarios included a standardized patient, a standardized nurse, and a part-task pelvis simulator (Postpartum bleeding station; ProDelphus, Olinda, Brazil). The trained domains comprised skills, knowledge, and attitudes tasks such as communication, teamwork, and clinical management.

The interview guide used open-ended questions to explore residents' perceived long-term transfer (Table 2) and was based on Baldwin and Ford's transfer model. ${ }^{18}$ According to this model, three factors influence transfer: (1) trainee characteristics (the motivation to attend training, the perceived relevance of the training content, and self-confidence); (2) the training design (application of ID principles); and (3) the workplace environment (organizational feedback, teamwork, and opportunity to use what has been learned). ${ }^{18,19}$

A fifth-year medical student was trained to conduct all one-to-one interviews in a private room in the hospital. The participants received two visual aids to facilitate recollection: the picture summarizing the PPH protocol (Fig. 1) and a picture summarizing the PPH simulation steps from each training format. ${ }^{15}$ The interviews were audio-recorded and typically lasted 20 minutes. They were transcribed verbatim and

TABLE 1 List of instructional design elements used in the instructional design simulation group.

\begin{tabular}{|c|c|c|}
\hline What & Concept & Application in the present study \\
\hline Authenticity ${ }^{9}$ & Real life, whole tasks & Scenario details very similar to reality; SKA tasks expected \\
\hline Psychological fidelity ${ }^{11}$ & $\begin{array}{l}\text { Degree to which real-task skill(s) are replicated in the } \\
\text { simulated task }\end{array}$ & $\begin{array}{l}\text { In situ environment, simulated patient and simulated nurse } \\
\text { contributed to the "suspension of disbelief" (acting as if } \\
\text { the scenario was real) }\end{array}$ \\
\hline Engineering fidelity ${ }^{11}$ & $\begin{array}{l}\text { Degree to which the training device or environment } \\
\text { replicates the physical characteristics of the real task }\end{array}$ & $\begin{array}{l}\text { Scenarios occurred in situ, with simulated patient and } \\
\text { interactive part-task simulator }\end{array}$ \\
\hline Paivio's dual coding ${ }^{12}$ & Visualization of instructions facilitates learning & $\begin{array}{l}\text { A circle with continuous arrows (Fig. 1) summarized the } \\
\text { training content }\end{array}$ \\
\hline Feedback $^{13}$ & Trainees receive information on their performance & $\begin{array}{l}\text { Trainees received feedback (debriefing steps) on more than } \\
\text { one occasion }\end{array}$ \\
\hline Variability $^{14}$ & $\begin{array}{l}\text { Opportunity to practice different tasks in more than } \\
\text { one scenario }\end{array}$ & Trainees attended three different scenarios \\
\hline Increasing complexity ${ }^{14}$ & $\begin{array}{l}\text { Learning tasks are presented in a sequence of } \\
\text { increasing complexity }\end{array}$ & $\begin{array}{l}\text { Scenarios were presented in a sequence of increasing } \\
\text { complexity }\end{array}$ \\
\hline
\end{tabular}

Abbreviation: SKA, skills, knowledge, and attitude. 


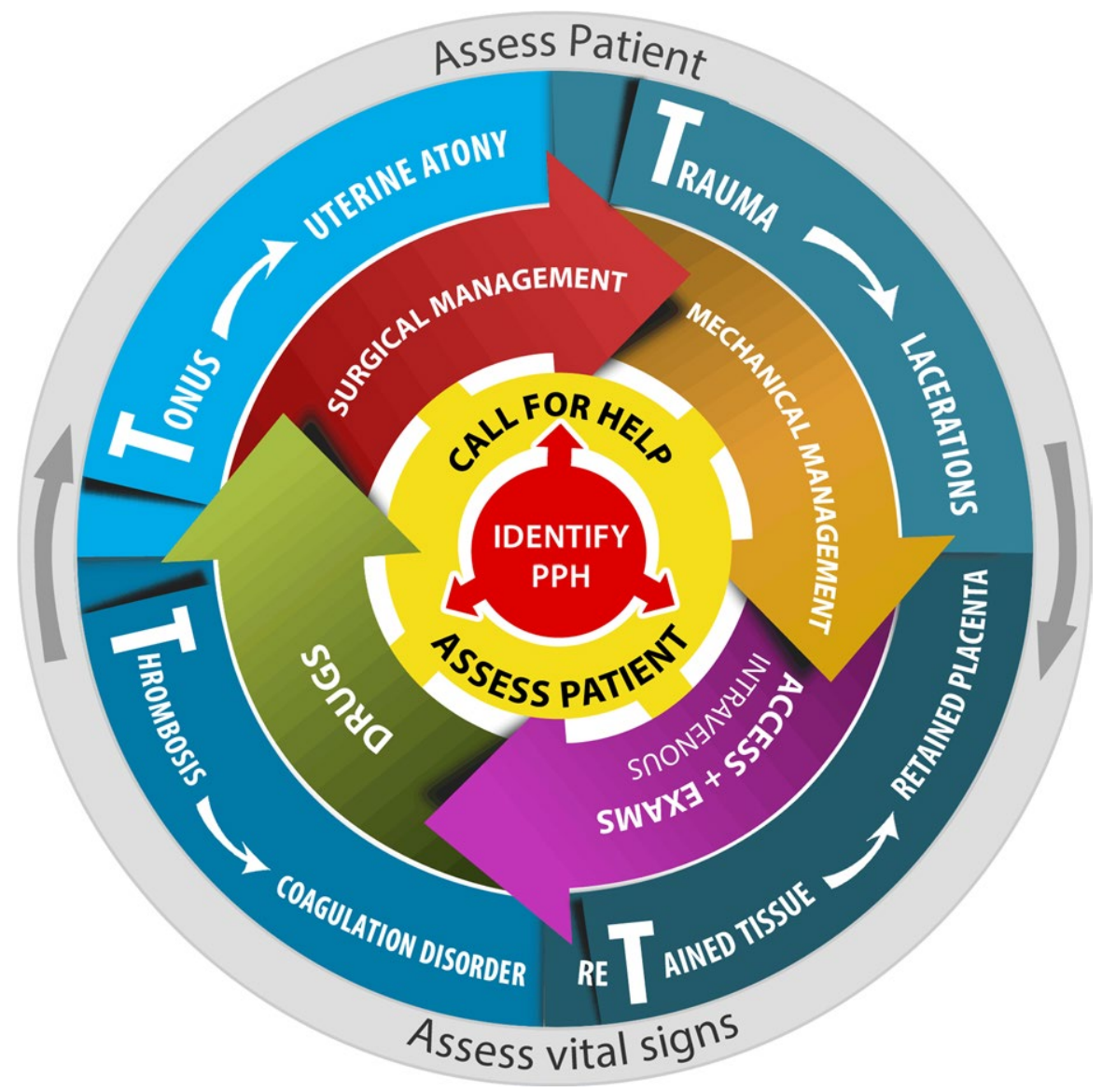

FIGURE 1 Postpartum hemorrhage management protocol.

TAB LE 2 Interview guide: list of questions for the semistructured questionnaire.

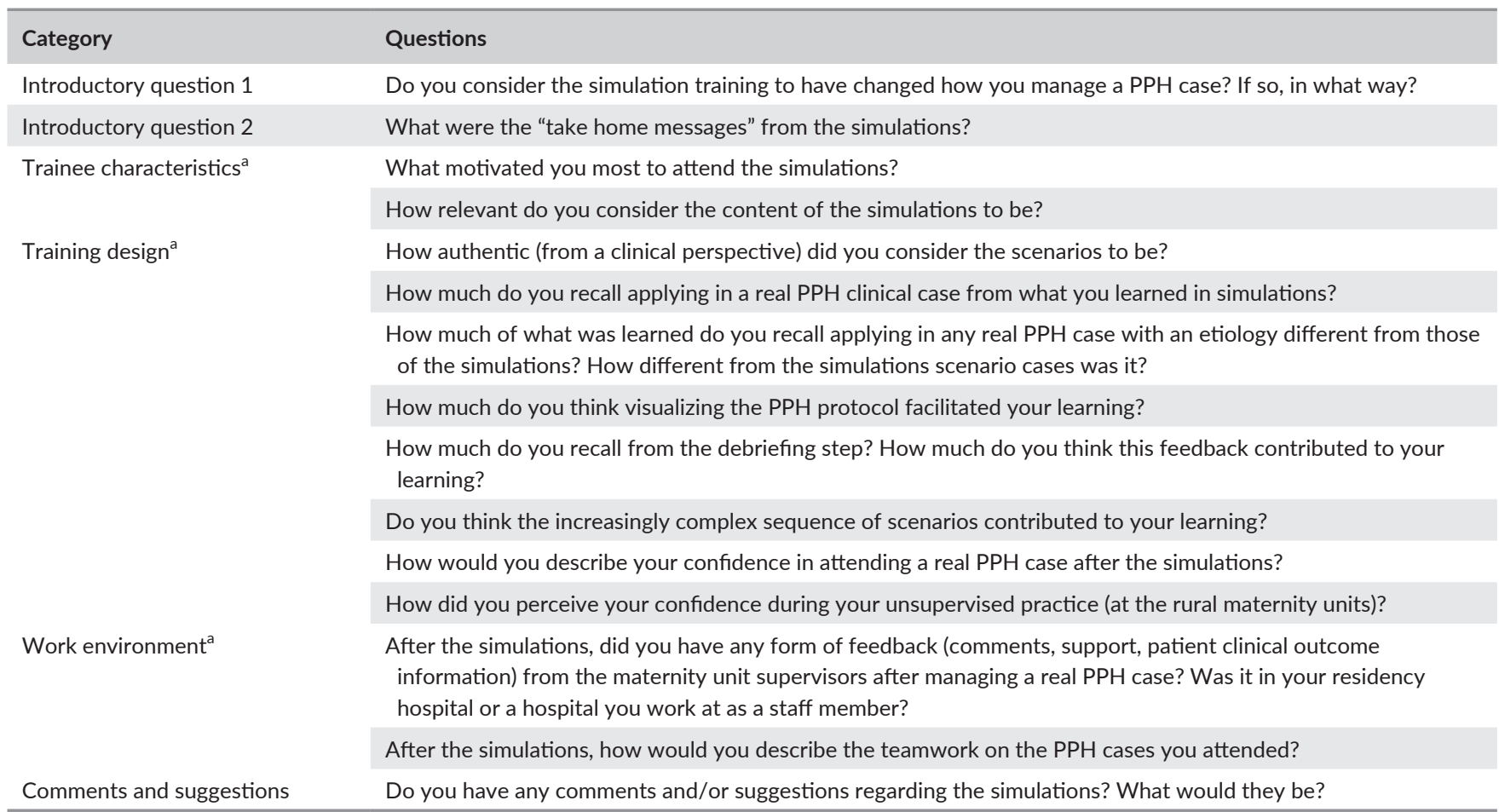

Abbreviation: PPH, postpartum hemorrhage.

${ }^{a}$ Baldwin and Ford's training transfer framework. ${ }^{17}$ 
the participants' identities were coded according to the format of the attended simulation (Resident_ID\# or Resident_BP\#).

Thematic analysis ${ }^{20}$ was used to analyze the interviews. Two researchers (BCPM and ARF), who were blinded to the participants' identity but not to the format of the attended simulation, independently read the transcripts of each interview and highlighted, labeled, and grouped relevant quotations in a coding table.

In a first discussion round, the readers compared the highlighted quotations and coding tables and agreed on two preconceived main categories: (1) perceived transfer and (2) simulation factors perceived as potentially affecting transfer. The simulation factors category was composed of three subcategories that were based on the model by Baldwin and Ford ${ }^{18}$ : (2a) trainee characteristics, (2b) simulation design, and (2c) work environment. One particular group of similar quotations did not fit into the preconceived main categories and was categorized as "systematic approach to PPH management". ${ }^{10}$

Subsequently, each reader independently created a table for each simulation format (BP or ID) and displayed the findings according to the main categories and subcategories. They then discussed differences in perceived long-term transfer per format. A final table containing the summary columns for each simulation format was translated into English for interpretation and discussion by the other authors. Saturation of the findings was achieved once the collected data allowed sufficient understanding of the dimensions and properties of our key concepts. ${ }^{21}$ No further sampling of residents was deemed necessary.

The English-language table contained quotations of the residents, organized by category, and notes from the readers. The notes described reflections regarding the residents' perception of overall transfer; the potential effects of different simulation factors (in particular ID elements) on transfer; and observed differences in perceived transfer depending on the simulation format.

\section{3 | RESULTS}

All 12 potential interviewees accepted the invitations. The mean age of the interviewees was 29 years (range 28-31 years), the mean time since graduation from medical school was 4 years (range $2.5-5$ years), and 11 interviewees were female.

The first research question was addressed by ascertaining whether residents from both simulation formats perceived long-term transfer effects. Residents consistently reported a perceived change in PPH management and the use of a more systematic approach to problemsolving after the simulations (Table 3).

The findings relating to the second research question (influence of simulation factors on transfer) were presented as follows: (a) trainee characteristics (relevance, motivation, and self-confidence), (b) training design (authenticity, dual coding, feedback by debriefing, and variability and increasing complexity), and (c) work environment (organizational feedback, teamwork, and opportunity to use what was learned).

The reported motivation for attending the simulations was a desire to learn and improve professional skills. The residents acknowledged that the content of the simulations (management of PPH) was relevant to them. Moreover, they perceived heightened self-confidence in the management of patients with PPH in their real work environment after the simulations, in particular when working in an unsupervised situation (outside their residency programs). The simulations gave them the confidence to assume team leadership when necessary (Table 3).

With regard to the training design, the scenarios were considered authentic in so far as they genuinely replicated clinical cases encountered in the residents' workplace practice, with similar reasoning challenges. Furthermore, the simulations occurred in situ (in a real environment) in the presence of a simulated nurse and a simulated patient. All these items contributed to the psychological fidelity (mental similarity to reality) of the simulations, reflected by a perceived sense of anxiety during the simulations (Table 3). The engineering fidelity (physical similarity) of the part-task simulator was also mentioned as a factor contributing to the authenticity (overall similarity to real cases) of the simulations.

The dual-coding strategy was also acknowledged as facilitating transfer. Residents strongly recollected the visual representation of the PPH protocol and specifically referred to the central circle with continuous arrows, which reflected the need to execute continuous and simultaneous tasks.

The residents reported that watching themselves on video during the debriefing (feedback) in both simulation formats allowed them to identify and reflect on their own errors and make plans for how to improve their skills. They also acknowledged that the safe feedback environment facilitated learning.

Residents who participated in the ID format were exposed to more than one training scenario and debriefing, which enabled them to try out variations and practice under conditions of increasing complexity. Residents attending this format reported that exposure to this training sequence facilitated learning (Table 3).

As for working-environment factors, organizational feedback was described as poor. With one exception, all residents said they had not received any workplace feedback (feedback from supervisors or institutional feedback).

The residents perceived an improvement in teamwork skills because they were both more aware of them after participating in the simulations and more motivated to take on a leadership role when faced with a real patient with $\mathrm{PPH}$. Residents who had managed a woman with PPH along with a colleague who had also attended the simulation stressed the smoothness of the actions and the shared responsibility in re-evaluating the diagnosis and revising the management plan. However, the residents also cited teamwork conflicts related to resistance from colleagues from other healthcare disciplines who had not attended the simulation training (Table 3).

With regard to the third research question, residents who had attended the ID-based simulation attributed the perceived transfer to improvements in both communication and teamwork skills, and also to situational awareness in the workplace. Moreover, only residents who had attended the ID simulation format reported communication with the bleeding woman and the collection of blood samples for examination early in the process, which reflects an awareness that the patient is potentially instable (increased situational awareness) (Table 3). 
TABLE 3 Extracted segments of interview responses.

Research question Quotations

First research question: How do participants perceive long-term transfer while performing on the job 2 years after attending the simulations (ID or BP)?

Perceived transfer

Resident_ID6: "What changed the most was the sequence of actions. What to start with: massage the uterus first, then go to medication, right? ... Acknowledging teamwork more, being aware of the need to call people to help, stabilize the patient, get a venous access, give her oxygen, monitor her vital signs... These were the things that really stuck in our minds after the training ... Now I have a more clearly defined sequence of actions ..."

Resident_BP4: "I think it was mostly, like... more speed in my reasoning! Bleeding: then I do this, then this, then that. Didn't work! Then this, afterwards that ..."

Second research question: Which factors of simulation do they perceive as positively affecting this long-term transfer awareness?

Trainee characteristics

Motivation and relevance of content

Resident_ID3: "If you work at a maternity, you have to practice. ... You need to have the young personnel trained to be aware of it"

Resident_BP6: "Very frequent. Managing it properly will save lives."

Self-confidence

Resident_ID5: "A lot, 100\%. You realize you are more confident, so you end up assuming more the leadership because of the training."

Simulation design

Authenticity

Resident_BP1: "For sure we deal with very similar cases in our day-to-day work"

Resident_ID1:"I recall I got very tense at that point, everybody got nervous. In this sense, it was very real."

Dual coding

Resident_ID1: "It is still in my mind today. I think it is great. I remember it every time. I recall the circle."

Resident_BP1: "It helps a lot. ... Because we can see it and recall the sequence very clearly."

Feedback

Resident_BP1: "I recall we were in a room watching what we had done. It was very useful. ... You can analyze what you did wrong, then you see what would have been right and you remember it."

Resident_BP1: "I remember well that the last case was much more complex and we managed it much faster than the initial (simpler) cases."

Increasing complexity (ID simulation only)

Resident_ID1: "It helped a lot. We were doing more and more increasingly more complex tasks."

Resident_ID3: "I think it [the increasing complexity of the scenarios] allowed us to gradually build up more knowledge"

Work environment

Feedback

Resident_BP1: "From a supervisor, once, at my residency. He congratulated me saying I knew how to take action, [how to] handle the case."

Teamwork and workplace learning skills

Resident_ID5: "There was this one case, I was with a colleague who had also attended the training. So things went very smoothly, we were thinking alike and getting feedback from each other. It was very interesting."

Resident_ID4: "The anesthesiologists are the ones who complicate our lives the most. I was very upset one day when they kind of neglected a case ... only when the patient began to become unstable did they realize it was for real."

Third research question: Are there differences in perceived long-term transfer according to the type of simulation attended (BP or ID)?

Differences in perceptions according to

Resident_ID4: "First is that thing of introducing yourself, explaining to the patient what is going on and your actions ... I didn't do that before. And collect exams, to remember to collect the exams ..."

Resident_ID2: "... already arriving at the scene talking to the patient, already managing the case, ... remembering to collect exams ..."

Abbreviations: BP, best practice; ID, instructional design; PPH, postpartum hemorrhage.

\section{DISCUSSION}

In the present study, residents perceived positive long-term transfer 2 years after having attended simulation training for $\mathrm{PPH}$, regardless of the simulation format (ID and BP). The reported motivation to attend the simulations was a desire to improve professional skills. The residents acknowledged that PPH was a relevant training topic and described an increase in self-confidence. Attendees of either simulation format 
reported the following ID elements as contributors to transfer: authenticity, ${ }^{9}$ use of Paivio's dual-coding strategy, ${ }^{12}$ and feedback during debriefing. ${ }^{14,22}$ Residents who participated in the ID-based simulation additionally mentioned variability and increasing complexity as ID elements contributing to transfer. Residents from both groups reported an increase in teamwork skills. They also reported poor organizational feedback and team struggles with colleagues who had not attended the simulation. Residents who had attended the ID format perceived better transfer of communication and teamwork skills and higher situational awareness than those having attended the BP format.

The present study makes several contributions to the transfer literature. Whereas most studies ${ }^{4,7}$ have explored transfer in the short term (immediately after training or a few weeks later), the present study demonstrated positive transfer 2 years after the PPH simulation training (long-term transfer). Moreover, the perceived improvements in communication skills and situational awareness reinforce the recommendation of applying ID principles when devising simulations, in particular simulations for high-stake situations such as PPH. ${ }^{1,5,6,10}$ In addition, because the study explored the perceptions of participants who, in their majority, had already concluded their residency, the study provides a wide range of perceptions deriving from actual workplace performance. This is likely to have had a positive influence on the perception of long-term training transfer. ${ }^{14}$

With regard to the influence of trainee characteristics, the motivation to improve one's professional skills has long been recognized as an important predictor for the effectiveness of transfer. ${ }^{4,19,23}$ The present study confirms this notion. The acknowledgement of PPH as a relevant topic for simulation training can be explained by the facts that PPH is a preventable cause of maternal death and that there has been a worrisome increase in the rate of $\mathrm{PPH}$, which has led to recommendations for healthcare personnel to participate in simulation training. ${ }^{1,2,17}$ The long time gap between training and the present evaluation of transfer reinforces the strength of the finding that self-confidence was increased. ${ }^{23}$ Whereas most previous studies have assessed self-confidence in the short term, ${ }^{4,7,19}$ residents in the present study were consulted after several on-the-job exposures to $\mathrm{PPH}$, which will have promoted better on-the-job learning. ${ }^{14}$

As for the training design, the following ID elements were reported as contributors to training transfer. First, authenticity, ${ }^{9,10}$ because the scenarios were close to reality and possessed psychological and engineering fidelity, which also contributed to the "suspension of disbelief" among the trainees. ${ }^{11}$ Although there is an ongoing debate as to the exact definition of authenticity, ${ }^{9-11,24}$ there is a general consensus that it is important for trainees to perceive scenarios as "challenging" and to feel motivated to exert effort ${ }^{19}$; this was indeed the case with the present scenarios. Second, the finding that visualization of the PPH protocol helped the residents to recall what had been learned reinforces Paivio's dual-coding theory. ${ }^{12}$ This might be of particular relevance to simulations of high-stake situations such as PPH, where several tasks have to be performed simultaneously and in collaboration. ${ }^{25}$ Third, feedback during debriefing is broadly recognized as being important for learning ${ }^{10,22}$; such feedback was provided more than once in the ID group. The residents stressed the importance of having the opportunity to learn from their own mistakes, a finding that is supported by evidence that error management improves transfer. ${ }^{4,25}$ Finally, the fact that attendees of the ID format acknowledged the variability and increasing complexity of the scenarios as contributors to transfer is in agreement with the literature on complex learning., ${ }^{4,14}$

When discussing the impact of the work environment (opportunity to use skills in practice, organizational feedback, teamwork skills) on transfer, the high prevalence of PPH ${ }^{1,17}$ is an important factor to consider. Given this high prevalence, the trainees had multiple on-thejob opportunities to apply the acquired skills, but it also emerged that poor organizational feedback was ubiquitous, ${ }^{4}$ highlighting the need to implement new policies for organizational feedback. With regard to teamwork, the perceived empowerment to take on a leadership role corroborates the need to train teamwork skills, preferably in a multidisciplinary fashion as illustrated by the reported conflicts with colleagues from other disciplines. ${ }^{25}$

The perception of enhanced communication skills and situational awareness by ID format residents reinforces the recommendation to apply ID principles when designing simulation training, particularly for high-stake situations such as PPH. The achievement of long-term transfer is particularly relevant to simulations for clinical conditions in which the optimal coordination of simultaneous tasks from multiple domains has an important impact on patient outcomes. ${ }^{1,2,14,25}$

The fact that the present analysis was based on self-perceived outcomes may be seen as a limitation. Indeed, strict analysis of transfer requires the overcoming of a few methodological challenges. For instance, to analyze PPH management in real life, uninterrupted video recording of all deliveries would be necessary (with all the accompanying costs and operational implications) because the occurrence of $\mathrm{PPH}$ is unpredictable. Another potential limitation is related to the fact that the ID and BP simulation formats contained a different number of scenarios. This reflects a core difference between the two formats: with the larger number of scenarios, the ID format provided multiple practice opportunities, variability, and increasing complexity, which promotes cognitive elaboration because learners are encouraged to compare and contrast scenarios with each other. In fact, these aspects reinforce the trustworthiness of the present findings, which were obtained 2 years after the actual simulations and after potential contamination from unrelated work experiences. ${ }^{10,14}$

In conclusion, the application of ID principles to PPH simulation training led to improvements in the perceived transfer of communication, teamwork, and situational awareness skills. Future studies should explore the contribution of specific ID elements to long-term transfer and their potential impact on clinical outcomes.

\section{AUTHOR CONTRIBUTIONS}

BCPM, JJGvM, and CvdV contributed to designing and planning the study, conducting the study, data analysis, and manuscript writing. ARF contributed to planning the study, conducting the study, data analysis, and manuscript writing. JLS contributed to data analysis and manuscript writing. 


\section{ACKNOWLEDGMENTS}

The authors acknowledge the support from Faculdade Pernambucana de Saúde and Instituto de Medicina Integral Professor Fernando Figueira. The authors would like to thank Ana Claudia Conrado de Oliveira for collecting interview data, and Bruno Hipólito and Daniel Ferreira for aiding with the visual representation of the PPH protocol and the editing of the video abstract.

\section{CONFLICTS OF INTEREST}

The authors have no conflicts of interest.

\section{REFERENCES}

1. World Health Organization. WHO Recommendations for the Prevention and Treatment of Postpartum Haemorrhage. Geneva, Switzerland: WHO Library Cataloguing-in-Publication Data; 2012.

2. Wong CA, Scott S, Jones RL, Walzer J, Geller S. The state of Illinois obstetric hemorrhage project: Pre-project and posttraining examination scores. J Matern Fetal Neonatal Med. 2016;29: 845-849.

3. Edozien LC. Situational awareness and its application in the delivery suite. Obstet Gynecol. 2015;125:65-69.

4. Salas E, Tannenbaum SI, Kraiger K, Smith-Jentsch KA. The science of training and development in organizations: What matters in practice. Psychol Sci Public Interest. 2012;13:74-101.

5. Cook DA, Hamstra SJ, Brydges R, et al. Comparative effectiveness of instructional design features in simulation-based education: Systematic review and meta-analysis. Med Teach. 2013;35:e867-e898.

6. McGaghie WC, Issenberg SB, Petrusa ER, Scalese RJ. A critical review of simulation-based medical education research: 2003-2009. Med Educ. 2010;44:50-63.

7. Mema B, Harris I. The barriers and facilitators to transfer of ultrasound-guided central venous line skills from simulation to practice: exploring perceptions of learners and supervisors. Teach Learn Med. 2016;28:115-124.

8. Burke LA, Hutchins HM. Training transfer: An integrative literature review. Hum Resource Dev Rev. 2007;6:263-296.

9. Merrill MD. First principles of instruction. Education Tech Research Dev. 2002;50:43-59.

10. van Merriënboer JJG, Kirschner P. Ten Steps to Complex Learning: A Systematic Approach to Four-component Instructional Design, 2nd edn. New York \& London: Routledge; 2013.
11. Maran NJ, Galvin RJ. Low- to high-fidelity simulation - a continuum of medical education? Med Educ. 2003;37:22-28.

12. Clark JM, Paivio A. Dual coding theory and education. Educ Psychol Rev. 1991;3:149-210.

13. Clark RE, Voogel A. Transfer of training principles of instructional design. ECTJ. 1985;33:113-123.

14. van Merriënboer JJG, Kester L, Paas F. Teaching complex rather than simple tasks: Balancing intrinsic and germane load to enhance transfer of learning. Appl Cogn Psychol. 2006;20:343-352.

15. de Melo BC, Falbo AR, Muijtjens AM, van der Vleuten $C P$, van Merriënboer JJ. The use of instructional design guidelines to increase effectiveness of postpartum hemorrhage simulation training. Int J Gynecol Obstet. 2017;137:99-105.

16. Clark EA, Fisher J, Arafeh J, Druzin M. Team training/simulation. Clin Obstet Gynecol. 2010;53:265-277.

17. Royal College of Obstetricians and Gynaecologists. Prevention and management of postpartum haemorrhage. RCOG Green-top Guidel. 2011;52:1-24.

18. Baldwin TT, Ford JK. Transfer of training: A review and directions for future research. Pers Psychol. 1988;41:63-105.

19. Blume BD, Ford JK, Baldwin TT, Huang JL. Transfer of training: A meta-analytic review. J Manag. 2010;36:1065-1105.

20. Braun V, Clarke V. Using thematic analysis in psychology. Qual Res Psychol. 2006;3:77-101.

21. Morse J. The significance of saturation. Qual Health Res. 1995;5: 147-149.

22. Fanning RM, Gaba DM. The role of debriefing in simulation-based learning. Simul Healthc. 2007;2:115-125.

23. Liaw SY, Scherpbier A, Rethans JJ, Klainin-Yobas P. Assessment for simulation learning outcomes: A comparison of knowledge and selfreported confidence with observed clinical performance. Nurse Educ Today. 2012;32:e35-e39.

24. Grierson LE. Information processing, specificity of practice, and the transfer of learning: Considerations for reconsidering fidelity. Adv Health Sci Educ Theory Pract. 2014;19:281-289.

25. Stocker $M$, Burmester $M$, Allen M. Optimisation of simulated team training through the application of learning theories: A debate for a conceptual framework. BMC Med Educ. 2014;14:1-9.

\section{SUPPORTING INFORMATION}

Additional Supporting Information may be found online in the supporting information tab for this article.

File S1. Portuguese translation of abstract. 